\title{
The type numbers of closed geodesics *
}

\author{
I.A. Taimanov ${ }^{\dagger}$
}

The seminal book [4] by M. Morse had put (together with the treatise by Lusternik and Schnirelmann [42]) the foundations for applying topological methods to variational problems.

Writing this appendix to its Russian translation we had no intention to survey wide applications of the Morse theory but wanted to complement the text by clarifications of some statements related to the type numbers of closed geodesics.

The foundations of what is called now the Morse theory together with their first applications are exposed in Chapters 1-7.

The last chapters 8 and 9 are concerned with applications of the Morse theory to the theory of closed geodesics.

In particular, the type numbers of closed geodesics in the space $\Omega$ of closed non-oriented curves without marked points are studied in Chapter 8. In [58] it was noted that the statement about these type numbers is not correct for iterated geodesics and the necessary correction is given.

In Chapter 9 this statement on the type numbers is used for computing the relative homology groups $H_{*}\left(\Omega\left(S^{n}\right), S^{n} ; \mathbb{Z}_{2}\right)$ for spheres (here $S^{n} \subset \Omega\left(S^{n}\right)$ is the set formed by one-point curves). Due to the mistake concerning the type numbers these computations are also wrong and these homologies are not computed until recently. However, the main problem which is studied in Chapter 9 is the existence of closed geodesics on manifolds sufficiently closed to ellipsoids. One of the main result proved by Morse is as follows:

- on an ellipsoid in $\mathbb{R}^{n+1}$ which is close to the round sphere but has pairwise different axes every prime closed geodesic is either one of $\frac{n(n+1)}{2}$ principal ellipses, either is very long.

${ }^{*}$ A translation of an appendix to the Russian edition of "Calculus of variations in the large" by M. Morse. The work was supported by RFBR (grant 09-01-00598) and Max Planck Institute for Mathematics in Bonn.

${ }^{\dagger}$ Institute of Mathematics, 630090 Novosibirsk, Russia; e-mail: taimanov@math.nsc.ru. 
The short modern proof of this theorem is given in 35. In the 1980s that was generalized by Ballmann [9] (for $n=2$ ) and then by Bangert [13] (for all $n$ ) who proved that

- for every $\varepsilon>0$ there exists $\delta=\delta(\varepsilon, n)$ such that every prime closed geodesic on a Riemannian sphere $S^{n}$ whose sectional curvature $K$ satisfies $1-\delta<K<1+\delta$ has length in $(2 \pi-\varepsilon, 2 \pi+\varepsilon)$ or larger than $\varepsilon^{-1}$.

Other results by Morse did also concern closed geodesics on spheres and were also formulated for metrics sufficiently closed to the round metrics. The modern approach to this problem was proposed by Ballmann, Thorbergsson, and Ziller who used the comparison theory and established the existence of simple closed geodesics for spheres with $\frac{1}{4}$-pinched metrics [12].

As we already mentioned the groups $H_{*}\left(\Omega\left(S^{n}\right), S^{n} ; \mathbb{Z}_{2}\right)$ are still not known however some nontrivial cycles were detected and were used for proving the existence of closed geodesics. This story did also involve some mistakes and "non-existing" cycles. The most recent description of these homologies is given in [4, 5].

The structure of our text is as follows.

In $\S \S 1-3$ we give a short introduction into the type numbers of closed geodesics and their applications and expose the necessary corrections to Morse'e results.

In $\S 4$ we demonstrate how these corrections were applied. Therewith we present the Bott formula for indices of iterated geodesics which is necessary for the demonstration and the Gromoll-Meyer theorem which is derived from the Bott formula.

Since all preliminary material is already done we could not resist a temptation to give in $\S 5$ a short survey of main methods and results concerning the application of the Morse theory [44] to proving the existence of closed geodesics.

In $\S 6$ we briefly remark on two active directions of developing the Morse theory and hope that this section shall invite researchers into the subject.

We thank D.V. Anosov, W. Ballmann, and H.-B. Rademacher for helpful discussions.

\section{The type numbers of closed geodesics}

In terms of relative homology groups the definition of type numbers [44, p.222] is as follows [59]. 
Let

$$
F: X \rightarrow \mathbb{R}
$$

be a smooth function. For arbitrary $r$ we put

$$
X_{r}=\{F<r\} \subset X, \quad \bar{X}_{r}=\{F \leq r\} .
$$

Let $c$ be a critical level of $f$ and $\omega \subset F^{-1}(c)$ be a set of critical points which probably does not contain all critical points with $F=c$. Let $U$ be a neighborhood of $\omega$ which does not contain other critical points than from $\omega$ and which is contractible to $\omega$ and let $\varepsilon>0$ be a positive constant such that the set

$$
X_{c} \backslash \bar{X}_{c-\varepsilon}=\{c-\varepsilon \leq F<c\}
$$

contains no critical points of $F$. Then the $k$-th type number $m_{k}$ of $\omega$ is the $k$-th Betti number of the pair $\left(X_{c-\varepsilon} \cup U, X_{c-\varepsilon}\right)$ :

$$
m_{k}(\omega)=\operatorname{rank} H_{k}\left(X_{c-\varepsilon} \cup U, X_{c-\varepsilon}\right) .
$$

This definition precedes Theorem 10.1 which is as follows:

- let $M_{k}$ be the sum of the $k$-th type numbers of all critical sets in $X_{a}$ and $B_{k}$ be the $k$-th Betti number of $X_{a}$, then

$$
\begin{gathered}
M_{0} \geq B_{0}, \\
M_{0}-M_{1} \leq B_{0}-B_{1}, \\
\cdots, \\
M_{0}-M_{1}+\cdots+(-1)^{r} M_{r}=B_{0}-B_{1}+\cdots+(-1)^{r} B_{r}
\end{gathered}
$$

for any sufficiently large integer $r$. 1

The inequalities (11) are well-known as the Morse inequalities and, in particular, they imply that

$$
M_{k} \geq B_{k},
$$

thus enabling us to prove the existence of critical points which are "topologically necessary". 2 The definition of the type numbers as well as of the Betti numbers depends on the choice of the coefficient group. Morse considered homologies with coefficients in $\mathbb{Z}$ and $\mathbb{Z}_{2}$.

\footnotetext{
${ }^{1}$ Here we assume that $X$ is compact or that $X_{a}$ contains finitely many connected critical sets $\omega$ for which all type numbers are finite and all of them except finitely many vanish.

${ }^{2}$ The Betti numbers are called the connectivities in [4].
} 
Closed geodesics on a Riemannian manifold $M$ are critical points of the length functional on the admissible spaces of closed curves: 3

$$
r:[0,1] \rightarrow M, \quad r(0)=r(1) .
$$

On this space acts the orientation-reversing map

$$
\sigma(r)=s \quad \text { if } r(t)=s(1-t) \text { for all } t .
$$

Since the length is independent of a parametrization we have to identify curves $r$ and $s$ if they are related by a change of parametrization:

$$
r \sim s \quad \text { iff } \quad r(t)=s(f(t)) \text { with } f:[0,1] \rightarrow[0,1] \text { and } f^{\prime} \geq 0 .
$$

Therewith we obtain the space $P^{+}(M)$ of oriented closed curves with a marked point which is $r(0)$. Any curve from $P^{+}(M)$ is represented uniquely by a map $r:[0,1] \rightarrow M$ with the length parameter $t$, i.e. $|\dot{r}|=$ const.

The quotient space

$$
P(M)=P^{+}(M) / \sigma
$$

is the space of non-oriented closed curves with a marked point.

On the space $P^{+}(M)$ there is a group action

$$
g_{\theta}: P^{+}(M) \rightarrow P^{+}(M), \quad \theta \in S^{1}=\mathbb{R} / \mathbb{Z},
$$

of $S^{1}=S O(2)$ which changes the marked points. Let $\gamma \in P^{+}(M)$ is represented by a map $r:[0,1] \rightarrow M$ with the length parameter $t$. Then $g_{\theta}(\gamma)$ is represented by

$$
r_{\theta}(t)=r((t+\theta) \bmod 1) .
$$

Since this action transforms closed closed into closed geodesics, to every nontrivial (i.e., not one-point curve) closed geodesic there corresponds a circle of critical points of the length functional.

By the involution $\sigma$ the action of $S O(2)$ is extended to the action of $O(2)$.

\footnotetext{
${ }^{3}$ Since the analytical aspects is not our main concern we avoid here rigorous definitions due to analytical complications. In fact, the length functional is not differentiable even on a smooth parameterized curve $r(t)$ such that $\dot{r}=0$ at some point. Hence, usually one have not only to restrict ourselves to curves for which the length is well-defined but to pass from spaces of such curves to their deformation retracts in which every closed geodesic line is represented and on which the length is defined as a smooth functional. This approach was applied by Morse.
} 
The periodic problem of the calculus of variations consists in estimating from below the number of closed extremals of functionals. In Riemannian geometry these extremals are geodesics.

To apply topological methods to finding closed geodesics we have to evaluate the number of critical points of the length functional on the admissible space of closed curves. Here we come to two problems:

1) in the space of non-parameterized curves with marked points to every closed geodesic line there corresponds an $S^{1}$-family of critical points;

2) in the quotient spaces

$$
\Omega^{+}(M)=P^{+}(M) / S O(2),
$$

the space of oriented closed curves (without marked points), and

$$
\Omega(M)=P^{+}(M) / O(2),
$$

to every nontrivial closed geodesic $\gamma$ there corresponds infinitely many critical points given by iterations of $\gamma$. So we say that 4

- a closed geodesic is prime if it is not a one-point geodesic or an iteration of another closed geodesic;

- a prime geodesic is called simple if it has no selfintersections;

- two closed geodesics are geometrically distinct if the corresponding geodesic lines on the manifold are different;

- two closed geodesics are called algebraically distinct if either they are geometrically distinct, either they are different iterations of the same prime closed geodesic.

Therefore different critical points of the length functional on

$$
F: \Omega^{+}=P^{+} / S^{1} \rightarrow \mathbb{R}
$$

correspond to algebraically distinct closed geodesics. The way to prove the existence of closed extremals is as follows:

\footnotetext{
${ }^{4}$ It is clear that these definitions are applicable to closed extremals of all functionals defined by some Lagrangian functions:$$
F(\gamma)=\int_{\gamma} L(x, \dot{x}) d t
$$

since for them iterations of extremals are also extremals. 

$M$;

1) to estimate the Betti numbers of $\Omega^{+}(M)$ in terms of the topology of

2) derive the existence of "topologically necessary" critical points of the length functional on $\Omega^{+}(M)$ from the Morse inequalities (1).

Remark. Another approach to look for closed geodesics, which is the most adopted now, is to consider the energy functional on the spaces of absolutely continuous closed curves with finite energy:

$$
\Lambda M=\left\{r: S^{1} \rightarrow M, r \text { is absolutely continuous, } \int|\dot{r}|^{2} d t<\infty\right\}
$$

(see [34]). This is the so-called free loop space and it is a Hilbert manifold. Such an approach has many nice features:

1) extremals of the energy functional on $\Lambda M$ are exactly length-parameterized geodesics;

2) any smooth map $f: M \rightarrow N$ induces a smooth map $\Lambda f: \Lambda M \rightarrow \Lambda N$ (if $f$ is only Lipschitz then $\Lambda f$ may be discontinuous):

3 ) the action of $O(2)$ is defined on $\Lambda M$ in the standard way and there is an equivariant continuous map $\Lambda M \rightarrow \Lambda M$ which is homotopical to the identity and maps $\Lambda M$ onto the subspace of length-parameterized curves. This implies that $\Lambda M, \Lambda M / S O(2)$ and their $\sigma$-quotients have the same homotopical properties as $P^{+}(M), \Omega^{+}(M)$ and their $\sigma$-quotients.

We refer to [3] for proofs of the statements 2) and 3).

Since the action of $S O(2)$ is not free (it branches exactly at iterated curves), $\Lambda M / S O(2)$ is "a Hilbert orbifold" and this causes serious difficulties.

\section{The finite-dimensional approximation of $P^{+}$}

We already mentioned in the footnote on p. 4that we have to deal with good models for the spaces $P^{+}$and $\Omega^{+}$, and now we recall one of the approaches which was used by Morse [44] (see also [43]).

Let us sketch his constructions and results.

Let $M$ be a compact Riemannian manifold. Let $\delta$ be a positive constant such that any two points $p, q \in M$ with $d(p, q) \leq \delta 5$ are connected by a unique geodesic $\gamma(p, q)$ with length $\leq \delta$ and moreover this geodesic smoothly depends on $p$ and $q$. For $M$ a compact manifold such a constant $\delta$ always exists and for it we can take any positive constant which is less than the injectivity radius.

\footnotetext{
${ }^{5}$ Here $d(p, q)$ is the distance function corresponding to the Riemannian metric on $M$.
} 
Let $P_{a}^{+}$be a space of closed curves with marked points and length less than $a$ and let

$$
N=\left[\frac{a}{\delta}\right]+1
$$

Starting from the marked point $r(0)$ divide every curve into $N$ successive pieces of equal length. If we take the length parameter on $r(t)$, then the separating points are

$$
r(0), r(1 / N), \ldots, r((N-1) / N), r(1)=r(0),
$$

Then let us replace every curve segment between $r(i / N)$ and $r((i+1) / N)$, $i=, \ldots, N-1$, by a unique shortest geodesic which connects these points, i.e. by $\gamma(r(i / N), r((i+1) / N))$. By construction, its length is less than $\delta$. Then the curve $r$ is replaced by a piecewise geodesic curve. All such geodesic polygons form a subset

$$
\Pi_{N} \subset \underbrace{M \times \cdot \times M}_{N}
$$

which is the closure of an open domain $U$ in the $N$-th power of $M$. The length functional gives rise to a smooth function $f$ on $U$, critical points of $f$ lie in $U$ and they are exactly closed geodesics. We have

$$
\operatorname{dim} U=N d, \quad \text { where } d=\operatorname{dim} M .
$$

The points in $\Pi_{N}$ are parameterized by sequences of the end-points by geodesic segments:

$$
\left(x_{0}, \ldots, x_{N-1}\right), \quad x_{i} \in M .
$$

In these terms the action of $\sigma$, the reversion of an orientation, reduces to

$$
\left(x_{0}, \ldots, x_{N-1}\right) \stackrel{\sigma}{\longrightarrow}\left(x_{N-1}, \ldots, x_{0}\right) .
$$

The group $S^{1}$ contains the subgroup $\mathbb{Z}_{N}$ which acts as follows

$$
\left(x_{0}, \ldots, x_{N-1}\right) \stackrel{\theta}{\longrightarrow}\left(x_{\theta}, \ldots, x_{(N-1+\theta) \bmod N}\right), \quad \theta \in \mathbb{Z}_{N} .
$$

The function $f$ is invariant with respect both to the $\mathbb{Z}_{N}$ action and to $\sigma$. Even after passing to $\Pi_{N} / \mathbb{Z}_{N}$ any nontrivial closed geodesic corresponds to an $S^{1}$-family of critical points of $f$.

However now we are in a finite-dimensional situation and can define the index and the nullity of a closed geodesic as of a critical point of smooth function. The index a closed geodesic was defined by Morse as follows. Let $x=\left(x_{0}, \ldots, x_{N-1}\right)$ corresponds to a closed geodesic $g$. At every point $x_{i}$ let 
us take an $(d-1)$-dimensional (i.e. a hypersurface) open submanifold $W_{i} \subset$ $M$ which passes through $x_{i}$ and transversal to the geodesic $g$. Assuming that $W_{i}$ are sufficiently small, we have an embedding

$$
W=W_{0} \times \ldots \times W_{N-1} \subset \Pi_{N} \subset M^{N} .
$$

The restriction of the length function onto $W$ is a smooth function

$$
f: W \rightarrow \mathbb{R}
$$

and we define the index and the nullity of the critical point $x$ as in a finitedimensional case:

- the index of $x$ equals the index of the Hessian of $f$ at $x$, i.e. the dimension of a tangent subspace at $x$ on which the Hessian of is negativelydefined;

- the nullity of $x$ is the difference of the dimensions of the tangent space in $x$ and its maximal subspace onto which the restriction of the Hessian is nondegenerate;

- a geodesic is nondegenerate if its nullity vanishes.

There is another way to define nondegenerate closed geodesics and their indices proposed by Bott [18]. Given a smooth function $f: X \rightarrow \mathbb{R}$ on a finite-dimensional manifold and a compact connected submanifold $Y \subset X$ which is a critical set of $f$, we say that $Y$ is nondegenerate if the nullity of $f$ at every point $x \in Y$ equals to the dimension of $Y$. The index of $Y$ is defined as the index of $f$ at $x \in Y$. Now it is said that a geodesic $g=x \in \Pi_{N}$ is nondegenerate if the corresponding $S^{1}$-family of critical points is nondegenerate in the Bott sense and the index of this geodesics is the index of the corresponding critical submanifold homeomorphic to $S^{1}$. 6

\footnotetext{
${ }^{6} \mathrm{~A}$ function for which all critical sets are nondegenerate is called a Morse-Bott function due to the Bott article [18] from 1954.

We have to stress that it was Pontryagin [52, 53] who at the first time considered such functions and even applied the extended Morse theory for solving a topological problem: a computation of the Betti numbers of classical Lie groups in 1935. In this case on every such a group there is a function with only two critical levels. For instance, given the group $S O(N)$ of orthogonal $N \times N$-matrices Pontryagin considered the entry $f=a_{11}$ in the top left corner of an orthogonal matrix as a smooth function on $S O(N)$ and proved that its only critical levels are \pm 1 and the corresponding critical sets are naturally diffeomorphic to $S O(N-1)$. Carefully analyzing how normal neighborhoods of critical sets are glued by the gradient flow of $f$ and starting with the simplest case $S O(3)=\mathbb{R} P^{3}$, Pontryagin
} 


\section{The type numbers of a closed geodesic in $\Omega^{+}$}

The sets of type (3) give only local finite-dimensional approximations of the space $\Omega^{+}=P^{+} / S^{1}$. The idea of Morse which is carefully discussed on $\mathrm{p}$. 249 is to use the limit of $\Pi_{N}$ as $N \rightarrow \infty$ for describing $\Omega^{+}$. If we have a geodesic polygon $x \in \Pi_{N}$ we may split every edge of it into $l$ parts of equal length. Thus we obtain a polygon with $N l$ edges and this canonical procedure gives us projections:

$$
\Pi_{N} \rightarrow \Pi_{N l} \text { for all } N, l .
$$

To these projections there corresponds a directed system of relative homology groups $H_{*}\left(\Pi_{N}, M\right)$ where by $M$ we denote the subset formed by trivial (one-point) curves. The $k$-th circular connectivity as it is defined by Morse [44] is the rank of the group

$$
\bar{p}_{k}=\operatorname{rank} \lim _{\rightarrow} H_{k}\left(\Pi_{N} / \sigma, M \mathbb{Z}_{2}\right),
$$

and the $k$-th sensed circular connectivity

$$
p_{k}=\operatorname{rank} \lim _{\rightarrow} H_{k}\left(\Pi_{N} ; \mathbb{Z}_{2}\right)
$$

was introduced by Bott [18]. Since, for any group of coefficients we have

$$
\lim _{\rightarrow} H_{i}\left(\Pi_{N}, M\right)=H_{i}\left(\Omega^{+}, M\right), \quad \lim _{\rightarrow} H_{i}\left(\Pi_{N} / \sigma, M\right)=H_{i}(\Omega, M)
$$

(see Theorem 9 in [58]), these topological invariants give lower estimates for sums of the type numbers of critical sets of the length functional, and hence imply the existence of nontrivial closed geodesics.

Morse stated that the type numbers $m_{k}$ (over $\mathbb{Q}$ or $\mathbb{Z}_{p}$ ) of a nondegenerate closed geodesic of index $i$ in $\Omega^{+}$(or $\Omega$ ) are equal to

$$
m_{k}=\delta_{k}^{i}
$$

[44, p. 296]. As it was first mentioned in [58] in general this is false however this is valid for prime closed geodesics. The failure of (4) is explained by the fact that

computed successively the $\bmod 2$ Betti numbers of all the groups $S O(N)$ and did the same for three other classical series.

However Pontryagin did not consider the general situation and did not define nondegenerate critical submanifolds and their indices. 
- the $S O(2)$ action is not free at iterated closed curves.

The right answer is given by the following 7

Theorem 1 ([58, [1]) Let $g$ be a prime closed geodesic, let $h=g^{s}$ be its $s$-th iteration, let $i(d)$ be the index of $g^{d}$, and let $d_{p}$ be the greatest divisor of $s$ which is not divided by a prime number $p$. We assume that the geodesic $g$ and its iterates are nondegenerate.

Then the type numbers $m_{k}$ and $m_{k}^{(p)}$ of $h=g^{s}$ in $\Omega^{+} \quad\left(\right.$ or in $\Omega=\Omega^{+} / \sigma$ ) with respect to coefficients in rational numbers and in $\mathbb{Z}_{p}$ take the form

1. if $s=1$, then

$$
m_{k}=m_{k}(p)=\delta_{k}^{i(1)}
$$

2. if $s \geq 2$, then

(a) if $s$ is odd or both $s$ and $i(2)-i(1)$ are even, then

$$
\begin{gathered}
m_{k}=\delta_{k}^{i(s)}, \\
m_{k}^{(p)}= \begin{cases}1 & \text { if } i\left(d_{p}\right)+2 \leq k \leq i(s), \\
0 & \text { otherwise }\end{cases}
\end{gathered}
$$

(b) if $s$ is even and $i(2)-i(1)$ is odd, then

$$
\begin{aligned}
& m_{k}=m_{k}^{(p)}=0 \quad \text { for all } k \text { and } p \neq 2, \\
& m_{k}^{(2)}= \begin{cases}1 & \text { if } i\left(d_{2}\right)+2 \leq k \leq i(s) \\
0 & \text { otherwise }\end{cases}
\end{aligned}
$$

The formula (44) was used in [44, 18] for computing mod 2 homologies of the spaces $\Omega$ and $\Omega^{+}$, respectively, for spheres. The failure of this fact explains why these computations were not correct and, to our knowledge, these homologies are still not known. 8

The rational cohomologies of these spaces were computed in [58]. We formulate these results in terms of the Poincare series

$$
p(X, t)=\sum_{k \geq 0} \operatorname{rank} H^{k}(X ; \mathbb{Q}) \cdot t^{k}, \quad p(X, Y, t)=\sum_{k \geq 0} \operatorname{rank} H^{k}(X, Y ; \mathbb{Q}) \cdot t^{k} .
$$

\footnotetext{
${ }^{7}$ The final corrected version of this result was given in [1].

${ }^{8}$ See the discussion in 31.
} 
Theorem 2 ([58]) For the spaces $\Omega^{+}\left(S^{n}\right)$ and $\Omega\left(S^{n}\right)$, we have

1. for $n$ even

$$
\begin{gathered}
p\left(\Omega^{+}, M, t\right)=t^{n-1}\left(\frac{1}{1-t^{2}}+\frac{t^{2 n-2}}{1-t^{2 n-2}}\right) \\
p(\Omega, M, t)=t^{n+1}\left(\frac{1}{1-t^{4}}+\frac{t^{2 n-4}}{1-t^{4 n-4}}\right)
\end{gathered}
$$

2. for $n \geq 3$ odd

$$
\begin{aligned}
& p\left(\Omega^{+}, M, t\right)=t^{n-1}\left(\frac{1}{1-t^{2}}+\frac{t^{n-1}}{1-t^{n-1}}\right) \\
& p(\Omega, M, t)=t^{n+1}\left(\frac{1}{1-t^{4}}+\frac{t^{n-3}}{1-t^{2 n-2}}\right)
\end{aligned}
$$

3.

$$
\begin{gathered}
p\left(\Omega^{+}, t\right)=1-t^{n+1}+p\left(\Omega^{+}, M, t\right), \\
p(\Omega, t)=1-t^{n+1}+p(\Omega, M, t)
\end{gathered}
$$

for all $n$.

For this computations Schwarz used the Leray-Serre spectral sequence applied to the homotopy quotients

$$
X_{S^{1}}=X \times_{S^{1}} E S^{1}, \quad X=P^{+},
$$

and their (homotopy) fibrations

$$
X_{G} \stackrel{X}{\longrightarrow} B G, \quad G=S^{1} .
$$

Since

$$
H^{*}\left(\Omega^{+} ; \mathbb{Q}\right)=H_{S^{1}}^{*}\left(P^{+} ; \mathbb{Q}\right),
$$

that enables us to compute the rational cohomologies of $\Omega^{+}=P^{+} / S^{1}$. This method was just introduced by Borel [17] and now

$$
H^{*}\left(X_{G}\right)=H_{G}^{*}(X)
$$

are called the equivariant cohomology of a space $X$ with $G$-action. Later $H^{*}\left(\Omega^{+}(M), M ; \mathbb{Q}\right)$ were computed by the same method for other compact rank one symmetric spaces $\mathbb{C} P^{n}, \mathbb{H} P^{n}$, and $\mathrm{Ca} P^{2}$ by Hingston 31] and, finally, Rademacher [54] did that for all spaces $M$ for which $H^{*}(M ; \mathbb{Q})$ is a truncated polynomial ring in one variable. 


\section{The Bott formula for the Morse indices of iter- ated closed geodesics and its applications}

Before demonstrating an important fact, i.e. Theorem 4, which was proved by using Theorems 1 and 2, we expose the Bott formula [19]. It plays a crucial role in proving the existence of a few closed geodesics and, especially, in the known proofs of the existence of infinitely many (geometrically distinct) closed geodesics on certain manifolds (see, in particular, Theorem 5). It is also necessary for proving Theorem 4 .

Theorem 3 ([19]) Let $g$ be a prime closed geodesic on a d-dimensional manifold $M$. Then there non-negative integer-valued functions $\Lambda_{g}$ and $N_{g}$ on the unit circle $|z|=1$ such that

1. $N_{g}(z)=\operatorname{dim} \operatorname{ker}\left(P_{g}-z\right)$ where $P_{g}$ is the Poincare map of the geodesic $g$ (it is given by $(d-1) \times(d-1)$-matrix);

2. $\Lambda_{g}(z)$ is constant at points at which $N_{g}(z)=0$ and its jumps are always bounded in absolute value by $N_{g}(z)$;

3. $\Lambda_{g}(z)=\Lambda_{g}(\bar{z}), N_{g}(z)=N_{g}(\bar{z})$;

4. the indices $i\left(g^{n}\right)$ and the nullities $\nu\left(g^{n}\right)$ of iterated geodesics $g^{n}$ are equal to

$$
i\left(g^{n}\right)=\sum \Lambda_{g}(\omega), \quad \nu\left(g^{n}\right)=\sum N_{g}(\omega),
$$

where $\omega$ ranges over the $n$-th roots of 1 if the parallel translation along $g$ does not change the orientation of $M$ and over the $n$-th roots of -1 overwise.

These formulas for indices and nullities are particular cases of facts concerning general self-adjoint differential equations with periodic coefficients.

A delicate corollary of Theorems 1 and 3, which is useful in applications, is as follows.

Lemma 1 ([23]) Let $g$ be a prime orientation-preserving closed geodesics of index $i(g)=l$ and let $g$ and all its iterations $g^{n}, n=1,2, \ldots$, are nondegenerate. Then for all $n$ we have

$$
m_{k}\left(g^{n}\right)=0 \quad \text { if } k-l \text { is an odd integer, }
$$

where $m_{k}$ are the type numbers in $\Omega^{+}$with respect to rational coefficients.

Hence, if $m_{k}\left(g^{l}\right) \neq 0$, then $k-i(g)$ is even. 
Proof. By Theorem 1

$$
m_{k}\left(g^{n}\right)= \begin{cases}\delta_{k}^{i\left(g^{n}\right)} & \text { when } i\left(g^{2}\right)-i(g) \text { is even } \\ 0 & \text { otherwise. }\end{cases}
$$

However, by Theorem 3 , if $i\left(g^{2}\right)-i(g)$ is even, then for all $n$ we have $i\left(g^{n}\right)-$ $i(g)$ is even and Lemma follows.

From Lemma 1 Fet derived the following theorem whose proof substantially uses Theorem 2, 9

Theorem 4 ([23]) Let $M$ be a simply-connected closed Riemannian manifold. Assume that all closed geodesics are nondegenerate. Then there are closed geodesics $g_{1}$ and $g_{2}$ with $m_{k}\left(g_{1}\right) \neq 0$ and $m_{k+1}\left(g_{2}\right) \neq 0$ with some $k<\operatorname{dim} M$. In particular, these geodesics are geometrically distinct.

The proof of Theorem 4 is based on the fact that for spheres we have

$$
H_{i}\left(\Omega\left(S^{n}\right), S^{n} ; \mathbb{Q}\right)=0 \quad \text { for all } i<n
$$

which, in its turn, is not true for the spaces of oriented curves:

$$
\operatorname{rank} H_{n-1}\left(\Omega^{+}\left(S^{n}\right), S^{n} ; \mathbb{Q}\right)=1 .
$$

One of the most substantial results which is derived by using the Bott formulas is the following theorem of Gromoll and Meyer:

Theorem 5 ([28]) Let $M$ be a simply-connected closed Riemannian manifold. If the Betti numbers of the free loop space $\Lambda M$ (for any given field of coefficients) are unbounded then there are infinitely many geometrically distinct (nontrivial) closed geodesics.

Since the Morse theory establishes a strong relation between critical points and the Betti numbers, for a proof Gromoll and Meyer did show that finitely many closed geodesics and their iterations can not generate the homology of the free loop space with unbounded Betti numbers.

More delicate consequences of the Bott formula were found and used by Rademacher (the average index formula [54]) and Long (the common index jump theorem [38]).

\footnotetext{
${ }^{9}$ In 23 it is claimed the existence of the second closed geodesic on a non-simplyconnected manifold is also proved but the arguments are wrong and this question is still open. This is explained on p. 15.
} 


\section{On lower estimates for the number of closed geodesics}

For completeness we expose here the main results concerning the existence of closed geodesics obtained by using variational methods and, in particular, by methods and ideas introduced by Morse [44].

We remark that whenever in this section we speak about estimates for the number of closed geodesics we mean prime closed geodesics.

\subsection{Geodesics on non-simply-connected manifolds}

For a nonsimply-connected manifold $M$ to every element $\tau \in\left[S^{1}, M\right]$, the set of free homotopy classes of maps from $S^{1}$ into $M$, there corresponds a connected component $P_{\tau}^{+}(M)$. Let $c_{\tau}$ be the infimum of the lengths of curves from $P_{\tau}^{+}(M)$ for some nontrivial class $\tau$. Then consider the finitedimensional approximation $\Pi_{N, \tau}$ of the subset $\{$ length $<c+\varepsilon\} \in P_{\tau}^{+}$, where $\varepsilon$ is any positive constant. On this finite-dimensional set the length reduces to a function which is smooth in the interior of $\Pi_{N, \tau}$. It is easy to show that this function admits its minimum at a smooth point which, by construction, is a closed geodesic $\gamma_{\tau}$ with minimal length in $\tau \in\left[S^{1}, M\right]$. Since $\tau$ is nontrivial, the geodesic $\gamma_{\tau}$ is also nontivial. This proves

Theorem 6 (Hilbert) On every non-simply-connected Riemannian closed manifold there exists a closed geodesic which is not homotopic to zero.

Now we come to the problem: how to prove that the geodesics which are minimal in the classes $\tau_{1}$ and $\tau_{2}$ are geometrically different?

We recall that

$$
\left[S^{1}, M\right]=\pi_{1} / \sim, \quad \text { where } x \sim y \text { if there is } z \text { such that } x=z y z^{-1}
$$

and $H_{1}(M)$ is the abelianization of $\pi_{1}$ :

$$
H_{1}=\pi_{1} /\left[\pi_{1}, \pi_{1}\right]
$$

Let

$$
H_{1}(M)=\mathbb{Z}+\mathbb{Z}+A
$$

where $A$ is some group and let $a$ and $b$ be the generators of the first two $\mathbb{Z}$ subgroups. Let us take the free homotopy classes $\tau_{p}$, where $p$ range over prime numbers, which represent the homology classes $a+p b$. It is easy to notice that the closed geodesics $\gamma_{\tau_{p}}$ are geometrically distinct. In particular, 
this argument shows that metrics on all closed oriented surfaces except $S^{2}$ have infinitely many closed geodesics. 10

Until recently for a general fundamental group (save of cyclic groups) it is not proved that the minimizers in different free homotopy class give us at least two geometrically distinct closed geodesics! In fact, we can not exclude the existence of a finitely-presented group $\pi$ with the following property:

- there is an element $a \in \pi$ such that every nontrivial element $b \in \pi$ is conjugate to some power of $a: a^{k} \sim b$.

At least it is known that such noncyclic finitely-generated groups do exist [30] (however all known such examples are not finitely-determined groups). This demonstrates a flaw in the arguments from [23]. Despite the claim from this article the existence of at least two closed geodesics on every nonsimply-connected closed manifold is not proved yet.

For seeking more closed geodesics we have to look for non-minimal closed geodesics. For proving their existence one can use higher homotopy groups of $\Omega_{\tau}^{+}(M)$ computed in [10, 61] in terms of action of the fundamental group on higher homotopy groups of $M$. Until recently the class of finitely-presented groups is not not well understood and that obstructs deriving very general statements on the existence of many closed geodesics on non-simplyconnected manifolds. 11

However for $\pi_{1}=\mathbb{Z}$ Bangert and Hingston by very nice arguments involving the actions of $\pi_{1}$ on $\pi_{n}, n \geq 2$, proved not only the existence of infinitely many closed geodesics but also the asymptotic formula for the growth of their lengths [15]. Let $N(t)$ be a number of geometrically distinct

\footnotetext{
${ }^{10}$ By passing to the universal covering, we may claim the same for all non-oriented surfaces except $\mathbb{R} P^{2}$. The cases of $S^{2}$ and $\mathbb{R} P^{2}$ are covered by Theorem 9 .

${ }^{11}$ We would like to mention another approach to study the existence of closed geodesics in which algorithmic properties of the fundamental group plays the main role. In 1976 Gromov 29] stated the following theorem whose proof was exposed in [45]:

- if the word problem for $\pi_{1}(M)$ is unsolvable, then on a closed manifold $M$ there exists an infinite family of prime contractible closed geodesics $g_{n}$ such that each of them provides local minimum for the length functional and length $g_{n} \rightarrow \infty$ as $n \rightarrow \infty$.

The proof shows that if there are only finitely many prime contractible closed geodesics then there is an algorithm to decide whether any word in $\pi_{1}$ is trivial or not.

Nabutovsky distinguished in terms of the Kolmogorov complexity of the word problem the class $K$ of groups such that for a closed manifold $M$ with $\pi_{1}(M) \in K$ there exists a constant $c=c(M)>1$ such that the number $N(t)$ of contractible closed geodesics of length $\leq t$ grows at least as $c^{t}$ [45].
} 
closed geodesics of length $\leq t$. Then

$$
N(t)>C \frac{t}{\log t}
$$

with $C$ a positive constant.

\subsection{Geodesics on simply-connected manifolds}

ON THE EXISTENCE OF A CLOSED GEODESIC.

The existence of a nontrivial closed geodesic on a sphere $S^{n}$ of any dimension $\geq 2$ was established by Birkhoff as follows.

Let us take a singular foliation of the sphere by circles and points. For a two-dimensional sphere $S^{2}$ realized in $\mathbb{R}^{3}$ as the unit sphere which foliation is formed by plane sections where planes are orthogonal to some fixed axis. Assume that these curves are oriented, then this family of closed curves on $S^{n}$ form an $(n-1)$-dimensional cycle $\beta_{n-1} \in P^{+}\left(S^{n}\right)$. Since the family is glued together into a manifold which is mapped onto $S^{n}$ with degree one, this cycle is nontrivial in $H_{n-1}\left(P^{+}\left(S^{n}\right) ; \mathbb{Q}\right)$. It generates also a cycle in $H_{n-1}\left(P\left(S^{n}\right) ; \mathbb{Z}\right)$. Since in non-oriented case the degree of the map is defined mod 2 this cycle may lie in 2-torsion in $H_{n-1}\left(P\left(S^{n}\right) ; \mathbb{Z}\right)$ and, in fact, that holds:

$$
2 \beta_{n-1}=0 \in H_{n-1}\left(P\left(S^{n}\right) ; \mathbb{Z}\right) .
$$

However from (11) it follows that $\beta_{n-1}$ suspends on some closed geodesic.

The existence of a nontrivial closed geodesic on any simply-connected closed manifold is derived by using these Birkhoff cycles. By the Hurewicz Theorem, for any simply-connected closed manifold $M$ there are nontrivial higher homotopy groups. In particular, if

$$
\pi_{1}(M)=0, \quad H_{i}(M)=0 \text { for } i<k \text { and } H_{k}(M) \neq 0,
$$

then

$$
\pi_{i}(M)=0 \text { for } i<k \text { and } \pi_{k}(M)=H_{k}(M) .
$$

Let us take a mapping $f: S^{k} \rightarrow M$ which realizes a nontrivial element $[f] \neq 0$ in $\pi_{k}(M)$. We take a singular foliation of $S^{k}$ and mapping it into $M$ we obtain the image $f_{*}\left(\beta_{k-1}\right) \in P^{+}(M)$ of the Birkhoff cycle. This cycle is also nontrivial, for the same reasons as the Birkhoff cycle, and on this cycle there suspends a closed geodesic.

Together with Theorem 6 this implies

Theorem 7 ([41]) On every closed Riemannian manifold there exists a closed geodesic. 
ON THE EXISTENCE OF INFINITELY MANY CLOSED GEODESICS.

Now there is a strong belief that on every, at least simply-connected, closed Riemannian manifold there are infinitely many (geometrically distinct) closed geodesics. A substantial progress in proving that was achieved in 1960-90s. We shall expose the main known results.

These results are proved mainly by applying the Morse inequalities (11) for establishing that the Betti numbers of $P(M)$ or $\Omega(M)$ can not be generated by finitely many nontrivial closed geodesics and their iterates. More complicated ring structure of the cohomologies of these spaces is not used. Other approaches were used only for two-dimensional manifolds (see the survey [63]).

The development of rational homotopy theory (minimal models by Sullivan) led to the following result by Sullivan and Vigué-Poirret:

Theorem 8 ([64]) If the rational cohomology ring of a closed simply-connected manifold $M$ is not a ring of truncated polynomials of one variable, then the rational Betti numbers of the free loop space $\Lambda M$ are unbounded.

Together with Theorem 5 this implies

Corollary 1 ([64]) If the rational cohomology ring of a closed simply-connected manifold $M$ has at least two multiplicative generators, then any Riemannian metric on $M$ has infinitely many closed geodesics.

So we left only with manifolds for which

$$
H^{*}(M ; \mathbb{Q})=\mathbb{Q}[u] / u^{d+1},
$$

which are, for instance, compact rank one symmetric spaces. For them the rational Betti numbers of $\Lambda M$ are bounded.

In early 1990s its was proved that

Theorem 9 ([14, 24]) Every Riemannian metric on a two-sphere has infinitely many closed geodesics.

Theorem 9 is proved by combining results of Bangert [14] and by Franks 24]. It needs to consider two cases: 1) there exists a simple closed geodesic for which the Birkhoff map is defined; 2) there is no such a geodesic. In the first case Franks proved the statement by using the theory of area-preserving diffeomorphisms which goes back to the Poincare-Birkhoff theorem, and in the second case Bangert showed that there exists a simple closed geodesic 
without conjugate points (when considered as geodesic defined for all times) and then used the Morse theory to derive from that the existence of infinitely many closed geodesics. In [32] the arguments by Franks were replaced by Morse-theoretical reasonings however it is still necessary for proof to consider two cases independently and the difference between them is formulated in dynamical terms.

For other manifolds the only known lower estimate which differs from just the existence of a closed geodesic was recently found for three-spheres:

Theorem 10 ([40]) Every Riemannian metric on a three-sphere has at least two closed geodesics.

ON LOWER ESTIMATES FOR THE NUMBER OF CLOSED GEODESICS OF GENERIC METRIC.

It was Morse who first started to consider metrics meeting some generic conditions. He considered metrics for which all closed geodesics are nondegenerate (these metrics are called now bumpy metrics). In this case for every closed geodesic only one of its type numbers (with respect rational coefficients) does not vanish. This property is $C^{r}$-generic for $2 \leq r \leq \infty$ : that was announced by Abraham in 1960s and the proof of that was given by Anosov in [5].

Theorem 4 gave the best known lower estimate for the number of closed geodesic of a bumpy metric which is valid for all simply-connected closed manifolds.

However one may consider other generic conditions.

The result by Klingenberg and Takens [36] reads that

- the condition 12 for a metric that either all closed geodesics are hyperbolic, 13 either there exists a nonhyperbolic closed geodesic of twist type 14 is a $C^{r}$-generic condition for $4 \leq r \leq \infty$.

Until recently there are no known examples of simply-connected closed Riemannian manifolds with only hyperbolic closed geodesics. However to prove the existence of infinitely many closed geodesics for metrics meeting the KT condition it is enough to consider manifolds with only hyperbolic geodesics.

\footnotetext{
${ }^{12} \mathrm{We}$ shall call it the $\mathrm{KT}$ condition.

${ }^{13}$ In particular, they are nondegenerate and for them index grows linearly: $i\left(g^{n}\right)=n i(g)$.

${ }^{14}$ In this case, by Moser's result, near this geodesic there exists infinitely many closed geodesics. The proof of that relates to the same theory as the result by Franks used in proving Theorem 9
} 
For non-simply-connected manifold that was done by Ballmann, Thorbergsson, and Ziller who proved that

Theorem 11 ([1]) Given a closed manifold $M$, if there is a nontrivial element $a \in \pi_{1}(M)$ such that $\left[a^{m}\right]=\left[a^{n}\right] \in\left[S^{1}, M\right]$ for some integers $m \neq n$, then for any metric on $M$ meeting the $K T$ condition there is a constant $C>0$ such that

$$
N(t)>C \frac{t}{\log t}
$$

where $N(t)$ is the number of geometrically distinct closed geodesics of length $\leq t$

The hypothesis of Theorem 11 is satisfied if $a \neq 1$ and $a$ is of finite order, i.e. for groups with torsion elements.

The proof of the existence of infinitely many closed geodesics in the situation of Theorem 11 is as follows. Let us assume that all geodesics are hyperbolic and let us take a minimal geodesic $g$ in class $[a]$. Then for all $l$ the geodesics $g^{l m}$ and $g^{l n}$ have index 0 and are freely homotopic to each other. Since they lie in a connected space to every such a pair $g^{l m}$ and $g^{l n}$ there corresponds a geodesic of index 1 which suspends on a one-cycle which joins $g^{l m}$ and $g^{l n}$. These geodesics of index 1 are prime since for hyperbolic geodesics $i\left(h^{n}\right)=n i(h)$.

The case of KT metrics on simply-connected manifolds was considered by Rademacher who computed the rational cohomologies of $\Omega^{+}(M)$ for all manifolds meeting (5) and showed that they can not be generated by finitely many hyperbolic geodesics and their iterates. For proving that he introduced and used the average index formula for closed geodesics.

Theorem 12 ([54]) Every metric on a simply-connected closed manifold $M$ which meets the KT condition has infinitely many closed geodesics.

Later Rademacher considered so-called strongly bumpy metrics which are defined as metrics for which all (non-trivial) closed geodesics are nondegenerate, the eigenvalues of the Poincare maps for them are simple and every finite set of the eigenvalues of norm one are algebraically independent. In 55] he proved that this condition is $C^{r}$-generic for $2 \leq r \leq \infty$ and by using the average index formula also proved that for such metrics on simply-connected manifolds there exist infinitely many closed geodesics. 


\section{$6 \quad$ Finsler and magnetic geodesics}

Since our task was mostly to complement the new edition of Morse's book by necessary comments, we did not write an extensive survey on applications of the Morse theory to closed geodesics. We also put off the theory by Lusternik and Schnirelmann who used different topological invariants (category, cup length) which now serve as very strong tools in nonlinear analysis and calculus of variations. However the proof of their main theorem was completed only recently [8] (see also 63]).

At the end we would like to mention briefly two active subjects where the Morse theory is being applied and where there are still a plenty of open problems.

\subsection{Finsler geodesics}

Although the study of closed geodesics for Finsler metrics was initiated by Anosov in the middle of 1970s 2] it became an active area of research only recently.

A Finsler metric $F(x, \dot{x})$ is a nonnegative function on the tangent bundle which is convex in $\dot{x}$ and meets the condition

$$
F(x, \lambda \dot{x})=\lambda F(x, \dot{x}) \quad \text { for } \lambda>0 \text {. }
$$

Riemannian metrics supply a particular class of Finsler metrics:

$$
F(x, \dot{x})=\sqrt{g_{i k}(x) \dot{x}^{i} \dot{x}^{k}}=|\dot{x}| .
$$

In fact, the whole Morse theory works for Finsler metrics without changes: it works for sufficiently general functionals on loop spaces and, in particular, to the Finsler length. However we have to distinguish between reversible and irreversible Finsler metrics: a Finsler metric is called reversible if

$$
F(x, \dot{x})=F(x,-\dot{x})
$$

If a metric is reversible, then the change of orientation of a closed curve preserves the Finsler length and we may deal with the space of non-oriented curves, i.e. with $P(M)$ and $\Omega(M)$. Otherwise, for irreversible metrics we have to look for critical points in $P^{+}(M)$ and $\Omega^{+}(M)$.

There are strong differences between the homologies of spaces of oriented and of spaces of un-oriented curves. For instance, the Birkhoff cycle $\beta_{n}$ is nontrivial in $H_{n}\left(P^{+}\left(S^{n+1}\right) ; \mathbb{Q}\right)$ and vanishes in $H_{n}\left(P\left(S^{n+1}\right) ; \mathbb{Q}\right)$. For 
instance, this explains why Theorem 4 does not hold for geodesics of irreversible metrics (the proof from [23] works only for reversible metrics and for irreversible metrics the counterexample is given by the Katok example of a Finsler metric on $S^{2}$ for which there are only two closed geodesics $g_{1}$ and $g_{2}$ with $m_{k}\left(g_{1}\right) \neq 0$ and $m_{k+1}\left(g_{2}\right) \neq 0$ [33]). However, the proofs of Theorems 5, 6, 17, 11, 12 and of Corollary 1] work without changes for all Finsler metrics.

In general we can not expect the existence of infinitely many closed Finsler geodesics. An example of Katok which we already mentioned shows that there is an irreversible bumpy Finsler metric on $S^{2}$ admitting only two prime closed geodesics which are given by the same curve passed in different directions [33, 65].

In the last years the periodic problem for Finsler geodesics as well the differential geometry in large of Finsler spaces attracts a lot of attention [16, [39, 56]. In particular, very recently Bangert and Long proved the existence of at least two geometrically distinct closed geodesics on every Finsler 2sphere [16] (this was claimed in [2] but the proof did never appear).

An excellent survey on the periodic problem in the Finsler geometry and a list of open problems is presented in [39].

\subsection{Magnetic geodesics}

A simple example of a Finsler metric is provided by the following function

$$
L(x, \dot{x})=|\dot{x}|+A_{i} \dot{x}^{i},
$$

where $A_{i} d x^{i}$ is a one-form. If $A$ is sufficiently small:

$$
L(x, \dot{x})>0 \quad \text { for } \dot{x} \neq 0,
$$

we have an irreversible Finsler metric which in the Finsler geometry is called a Randers metric and we may apply the classical Morse theory.

We note that $A$ comes into the corresponding Euler-Lagrange equations via its differential

$$
F=\sum_{i<k} F_{i k} d x^{i} \wedge d x^{k}, \quad F_{i k}=\frac{\partial A_{k}}{\partial x^{i}}-\frac{\partial A_{i}}{\partial x^{k}},
$$

and that is easily explained by physics where solutions of the Euler-Lagrange equations for the Lagrangian

$$
L(x, \dot{x})=\sqrt{E g_{i k} \dot{x}^{i} \dot{x}^{k}}+A_{i} \dot{x}^{i}
$$


are (up to a parameterization) trajectories of a charged particle in a magnetic field on a fixed energy level $E$, where the Riemannian metric determines the kinetic energy, and a closed two-form $F$ is a magnetic field [49]. Hence for physical reasons only $F$ has to be globally defined and if $F$ is not an exact form, then the one-form $A$ is defined only locally. So we have two cases which are drastically different from periodic problems considered before by the Morse theory:

a) the form $A$ is defined only locally, in this case the formula

$$
S(\gamma)=\int_{\gamma}\left(|\dot{x}|+A_{i}^{\alpha} \dot{x}^{i}\right) d t
$$

determines a multi-valued functional on the space of closed curves (for instance, on $P^{+}(M)$ ) with a uniquely and globally defined variational derivative $\delta S$ (here $A_{i}^{\alpha} d x^{i}$ is the form $d^{-1} F$ defined in some domain $U_{\alpha}$ which contains $\gamma$ and for which the second Betti number vanishes);

b) even if $A$ is globally defined, then the functional (6) may be not bounded from below. In this situation when using the Morse theory we have to consider the homology groups $H_{*}\left(P^{+}(M),\{S \leq 0\}\right)$ instead of $H_{*}\left(P^{+}(M), M\right)$ in the classical Morse theory.

The extremals of the functional (6) are called magnetic geodesics. Sometimes the flow of such extremals is called twisted geodesic flow however we do not use this term and prefer to stress the physical origin of the subject.

To the functional (6) one may apply the whole local part of the Morse theory, defining the index and the nullity of extremals.

The study of the existence of critical points of these functionals as well as developing the analog of the Morse theory for one-forms (multivalued functions with globally defined differential) was started by Novikov in 1980 [46, 47, 48, 49]. The finite-dimensional theory was far-developed [22, 51] and its main counterparts such as the Morse-Novikov inequalities and the Novikov ring found many applications. The ideas concerning multi-valued functions found applications in mathematical physics. Speaking about closed extremals in magnetic fields, we note that topological problems concerning the lower estimates for the number of critical levels were also basically solved (Novikov's principle of "throwing out cycles") [49, 60, 50]. However the main difficulty appeared in the analytical part.

For deriving the existence of critical points from the Morse inequalities (11) it needs to have a good deformation which decreases the value of $S$ at noncritical points and lets a nontrivial relative cycle to suspend on a critical point of $S$ in the limit. For the length functional Morse uses the following 
deformation on $\Pi_{N}$ : given a geodesic $N$-gon $\gamma=\left(x_{0}, \ldots, x_{N-1}\right) \in \Pi_{N}$, let us take the middle points $\left(q_{0}, \ldots, q_{N-1}\right)$ of its edges and transform $\gamma$ into another $N$-gon $T(\gamma)$ :

$$
\gamma=\left(x_{0}, \ldots, x_{N-1}\right) \stackrel{T}{\longrightarrow}\left(q_{0}, \ldots, q_{N-1}\right) \in \Pi_{N} .
$$

The deformation $T: \Pi_{N} \rightarrow \Pi_{N}$ is continuous and decreases the lengths of curves except geodesics. However the values of the one-valued functional (6) under an analogous deformation may grow: $S(T(\gamma))>S(\gamma)$, and in the limit a nontrivial relative cycle $\xi \in H_{*}(\{S<c+\varepsilon\},\{S<c-\varepsilon\})$ may suspend on "a critical point at infinity" lying on the critical level $S=c$ (see discussion in 63] $) .15$

This difficulty with the convergence of gradient deformations hinders the application of variational methods to this problem. The only general statement proved by variational methods for higher dimensions was obtained in [7] under some geometrical restrictions on the Riemannian metric and the magnetic field.

More results were obtained in the two-dimensional situation [63, 27]. In particular, the existence of a simple locally minimal closed magnetic geodesic was proved for "strong" magnetic fields on two-dimensional compact manifolds [50, 62].

In [20] for "non-strong" exact magnetic fields 16 on two-dimensional compact surfaces it was proved that a closed nontrivial extremal always exists. This was done by using the theory of the Mané critical level and the classical Morse theory (in the situation of Finsler metrics) and together with the existence result for "strong" magnetic fields this implies the existence of a closed magnetic geodesic on every energy level for an exact magnetic field on a closed two-dimensional manifold. 17

There is another approach initiated by Arnold [6] and Kozlov [37] to the periodic problem for magnetic geodesics and it is based on the fixed point theorems from symplectic topology (we refer to a recent article [26] which, in particular, contains a good survey of the results obtained via this approach).

\footnotetext{
${ }^{15}$ In the case of such a lack of compactness it is said that $S$ does not satisfy the PalaisSmale conditions for applying the Morse theory in the infinite-dimensional situation.

${ }^{16} \mathrm{~A}$ magnetic field $F$ is called exact if the two-form $F$ is exact: $[F]=0 \in H^{2}(M ; \mathbb{R})$.

${ }^{17}$ Again by a combination of different methods (symplectic and variational) it was recently proved that on almost all energy levels there exists a closed magnetic geodesic for an exact magnetic field [21]. One of the main ingredients of the proof is the result which states that if an energy level of a magnetic geodesic flow is of contact type then the (free time) action functional meets the Palais-Smale condition.
} 
A completely new approach to establish the existence of periodic magnetic geodesics on the two-sphere was very recently proposed in [57].

We would like to stress that the theory of periodic magnetic geodesics is still rich by open problems waiting for their solutions:

a) for the case of exact magnetic field the existence of a closed magnetic geodesic in general case is still an open problem;

b) for non-exact fields the existence result in general does not hold: there are no closed magnetic geodesics on a hyperbolic closed two-surface with its area form as a magnetic field [25]. However it is interesting to understand how generic is this phenomenon and under which conditions periodic trajectories exist.

\section{References}

[1] Al'ber, S.I.: Topology of function spaces. Soviet Math. Dokl. 7 (1966), 700-704.

[2] Anosov, D.V.: Geodesics in Finsler geometry, in: Proc. I.C.M., Vancouver, B.C., 1974, vol. 2, Montreal, 1975, pp. 293-297 (in Russian), Amer. Math. Soc. Transl. 109 (1977) 81-85.

[3] Anosov, D.V.: Some homotopies in a space of closed curves. Math. USSR-Izv. 17 (1981), 423-453.

[4] Anosov, D.V.: Homology in the space of closed curves on an $n$ dimensional sphere. Math. USSR-Izv. 18 (1982), 403-422.

[5] Anosov, D.V.: Generic properties of closed geodesics. Math. USSRIzv. 21 (1983), 1-29.

[6] Arnold, V.I.: First steps in symplectic topology. Russian Math. Surveys 41:6 (1986), 1-21.

[7] Bahri, A., and Taimanov, I.A.: Periodic orbits in magnetic fields and Ricci curvature of Lagrangian systems. Trans. Amer. Math. Soc. 350 (1998), 2697-2717.

[8] Ballmann, W.: Der Satz von Lusternik-Schnirelmann, Bonner Math. Schriften 102 (1978).

[9] Ballmann, W.: On the lengths of closed geodesics on convex surfaces. Invent. Math. 71 (1983), 593-597. 
[10] Ballmann, W.: Geschlossene Geodätische auf Mannigfaltigkeiten mit unendlicher Fundamentalgruppe. Topology 25:1 (1986), 55-69.

[11] Ballmann, W., Thorbergsson, G., and Ziller, W.: Closed geodesics and the fundamental group. Duke Math. J. 48 (1981), 585-588.

[12] Ballmann, W., Thorbergsson, G., and Ziller, W.: Existence of closed geodesics on positively curved manifolds. J. Differential Geom. 18 (1983), 221-252.

[13] Bangert, V.: On the lengths of closed geodesics on almost round spheres. Math. Z. 191 (1986), 549-558.

[14] Bangert, V.: On the existence of closed geodesics on two-spheres. Internat. J. Math. 4 (1993), 1-10.

[15] Bangert, V., and Hingston, N.: Closed geodesics on manifolds with infinite abelian fundamental group. J. Differential Geom. 19 (1984), $277-282$.

[16] Bangert, V., and Long, Yiming: The existence of two closed geodesics on every Finsler 2-sphere. Math. Ann. 346 (2010), 335366 .

[17] Borel, A.: Sur la cohomologie des espaces fibrés principaux et des espaces homogènes de groupes de Lie compacts. Ann. of Math. (2) 57 (1953), 115-207.

[18] Bott, R.: Nondegenerate critical manifolds. Ann. of Math. (2) 60 (1954), 248-261.

[19] Bott, R.: On the iteration of closed geodesics and the Sturm intersection theory. Comm. Pure Appl. Math. 9 (1956), 171-206.

[20] Contreras, G., Macarini, L., and Paternain, G.: Periodic orbits for exact magnetic flows on surfaces. Int. Math. Res. Not. 2004, no. 8, $361-387$.

[21] Contreras, G.: The Palais-Smale condition on contact type energy levels for convex Lagrangian systems. Calc. Var. Partial Differential Equations 27 (2006), 321-395.

[22] Farber, M.: Topology of closed one-forms. Mathematical Surveys and Monographs, 108. American Mathematical Society, Providence, RI, 2004. 
[23] Fet, A.I.: On a periodicity problem in the calculus of variations. Soviet Math. Dokl. 6 (1965), 85-88.

[24] Franks, J.: Geodesics on $S^{2}$ and periodic points of annulus homeomorphisms. Invent. Math. 108 (1992), 403-418.

[25] Ginzburg, V.L.: On the existence and non-existence of closed trajectories for some Hamiltonian flows. Math. Z. 223 (1996), 397-409.

[26] Ginzburg V., and Gürel, B.: Periodic orbits of twisted geodesic flows and the Weinstein-Moser theorem. Comment. Math. Helv. 184 (2009), 865-907.

[27] Grinevich, P.G., and Novikov, S.P.: Nonselfintersecting magnetic orbits on the plane. Proof of the overthrowing of cycles principle. Topics in topology and mathematical physics, 59-82, Amer. Math. Soc. Transl. Ser. 2, 170, Amer. Math. Soc., Providence, RI, 1995.

[28] Gromoll, D., and Meyer, W.: Periodic geodesics on compact riemannian manifolds. J. Differential Geometry 3 (1969), 493-510.

[29] Gromov, M.: Three remarks on geodesic dynamics and fundamental group. Enseign. Math. (2) 46 (2000), 391-402.

[30] Guba, V.S.: A finitely generated complete group. Math. USSR-Izv. 29 (1987), 233-277.

[31] Hingston, N.: Equivariant Morse theory and closed geodesics. J. Differential Geom. 19 (1984), 85-116.

[32] Hingston, N.: On the growth of the number of closed geodesics on the two-sphere. Internat. Math. Res. Notices 1993, no. 9, 253-262.

[33] Katok, A.B.: Ergodic perturbations of degenerate integrable Hamiltonian systems. Math. USSR-Izv. 7 (1973), 535-572.

[34] Klingenberg, W.: Lectures on closed geodesics. Grundlehren der Mathematischen Wissenschaften, 230, Springer-Verlag, BerlinNew York, 1978.

[35] Klingenberg, W.: Riemannian geometry. de Gruyter Studies in Mathematics, 1. Walter de Gruyter and Co., Berlin-New York, 1982. 
[36] Klingenberg, W., and Takens, F.: Generic properties of geodesic flows. Math. Ann. 197 (1972), 323-334.

[37] Kozlov, V.V.: Calculus of variations in the large and classical mechanics. Russian Math. Surveys 40:2 (1985), 37-71.

[38] Long, Yiming, and Zhu, Chaofeng: Closed characteristics on compact convex hypersurfaces in $\mathbb{R}^{2 n}$. Ann. of Math. (2) 155 (2002), $317-368$.

[39] Long, Yiming: Multiplicity and stability of closed geodesics on Finsler 2-spheres. J. Eur. Math. Soc. (JEMS) 8 (2006), 341-353.

[40] Long, Yiming, and Duan, Huagui: Multiple closed geodesics on 3-spheres. Adv. Math. 221 (2009), 1757-1803.

[41] Lusternik, L.A., and Fet, A.I.: Variational problems on closed manifolds. (Russian) Doklady Akad. Nauk SSSR (N.S.) 81 (1951). 1718.

[42] Lusternik, L.A., and Schnirelmann, L.G.: Topological methods in variational problems. (Russian) Proc. Mathematics and Mechanics Research Institute Moscow State Univeristy, GITTL, Moscow, 1930 .

[43] Milnor, J.: Morse theory, Ann. of Math. Studies, 51, Princeton Univ. Press, Princeton, NJ, 1963.

[44] Morse, M.: The calculus of variations in the large. AMS, Providence, RI, 1934.

[45] Nabutovsky, A.: Fundamental group and contractible closed geodesics. Comm. Pure Appl. Math. 49 (1996), 1257-1270.

[46] Novikov, S.P.: Multivalued functions and functionals. An analogue of the Morse theory. Soviet Math. Dokl. 24 (1981), 222-226.

[47] Novikov, S.P., and Shmel'tser, I.: Periodic solutions of Kirchhoff equations for the free motion of a rigid body in a fluid and the extended Lyusternik-Shnirel'man-Morse theory.I. Functional Anal. Appl. 15:3 (1981), 197-207.

[48] Novikov, S.P.: Variational methods and periodic solutions of equations of Kirchhoff type.II. Functional Anal. Appl. 15:4 (1981), 263274. 
[49] Novikov, S.P.: The Hamiltonian formalism and a multivalued analogue of Morse theory. Russian Math. Surveys 37:5 (1982), 1-56.

[50] Novikov, S.P., and Taimanov, I.A.: Periodic extremals of multivalued or not everywhere positive functionals. Soviet Math. Dokl. 29 (1984), 18-20.

[51] Pajitnov, A.V.: Circle-valued Morse theory. de Gruyter Studies in Mathematics, 32. Walter de Gruyter and Co., Berlin, 2006.

[52] Pontryagin, L.S.: Sur les nombres de Betti des groupes de Lie. C. R. Acad. Sci. Paris 200 (1935), 1277-1280.

[53] Pontryagin, L.S.: Homologies in compact Lie groups. Rec. Math. N. S. [Mat. Sbornik] 6 (48) (1939), 389-422.

[54] Rademacher, H.-B.: On the average indices of closed geodesics. J. Differential Geom. 29:1 (1989), 65-83.

[55] Rademacher, H.-B.: On a generic property of geodesic flows. Math. Ann. 298 (1994), 101-116.

[56] Rademacher H.-B.: A sphere theorem for non-reversible Finsler metrics. Math. Ann. 328 (2004), 373-387.

[57] Schneider, M.: Closed magnetic geodesics on $S^{2}$. arXiv:0808.4038, 2008.

[58] Schwarz, A.S.: The homologies of spaces of closed curves. (Russian) Trudy Moskov. Mat. Ob. 9, 1960, 3-44.

[59] Seifert, H., and Threlfall, W.: Variationsrechnung im Grossen (Theorie von Marston Morse). Leipzig and Berlin, Teubner, 1938.

[60] Taimanov, I.A.: The principle of throwing out cycles in MorseNovikov theory. Soviet Math. Dokl. 27 (1983), 43-46.

[61] Taimanov, I.A.: Closed geodesics on non-simply-connected manifolds. Russian Math. Surveys 40:6 (1985), 143-144.

[62] Taimanov, I.A.: Non-self-intersecting closed extremals of multivalued or not-everywhere-positive functionals. Math. USSR-Izv. 38 (1992), 359-374. 
[63] Taimanov, I.A.: Closed extremals on two-dimensional manifolds. Russian Math. Surveys 47:2 (1992), 163-211.

[64] Vigué-Poirrier, M., and Sullivan, D.: The homology theory of the closed geodesic problem. J. Differential Geometry 11:4 (1976), 633644 .

[65] Ziller, W.: Geometry of the Katok examples. Ergodic Theory Dynam. Systems 3 (1983), 135-157. 